Published in final edited form as:

Ophthal Plast Reconstr Surg. 2014 ; 30(1): . doi:10.1097/IOP.0b013e3182873d4c.

\title{
Osseous Metaplasia of the Eyelid: Report of Two Cases
}

\author{
Abby Y. Liu, BS ${ }^{1}$, Harvey P. Cole, MD², Melissa L. Meldrum-Aaberg, MD ${ }^{3}$, and Hans E. \\ Grossniklaus, MD ${ }^{1}$ \\ ${ }^{1}$ Department of Ophthalmology, Emory University School of Medicine \\ ${ }^{2}$ Oculus Plastic Surgery, Atlanta, Georgia \\ ${ }^{3}$ Eye Plastic \& Facial Cosmetic Surgery, Grand Rapids, Michigan
}

\section{Abstract}

Purpose-To describe two unusual cases of osseous metaplasia occurring within the eyelid and to present a brief review of the literature on cutaneous calcification.

Methods-Case reports and literature review.

Results-A 62-year old man presented with an eyelid mass containing osseous metaplasia in an area of lymphoid hyperplasia. An extensive oncologic workup ruled out malignancy. A 46-year old man with a history of recurrent chalazion presented with a firm mass of the upper eyelid that demonstrated bone formation in the area of a metaplastic sebaceous gland. Both lesions were excised with no further recurrence.

Conclusions-Osseous metaplasia of the eyelid is a rare entity with diverse etiologies ranging from congenital syndromes to trauma, neoplasm, and inflammation. In some cases, a precise etiology cannot be identified.

Osseous metaplasia is a rare phenomenon that occurs primarily in breast tissue and abdominopelvic organs. To date, there have been no previous reports of osseous metaplasia occurring within the eyelid. Herein we describe two patients with this unusual entity, and present a brief review of the literature concerning cutaneous ossification. Procedures followed were in accordance with the ethical standards of the responsible committee on human experimentation and with the Helsinki Declaration of 1975, as revised in 2000 and were HIPPA compliant.

\section{CASE REPORTS}

The first patient was a 62-year old male with a painless, mobile mass of the right upper eyelid measuring $6 \times 4 \mathrm{~mm}$ that had been enlarging over the course of three months (Figure 1). His past medical history was unremarkable. The mass was excised and sent for routine pathologic processing, where it was identified as reactive lymphoid hyperplasia with osseous metaplasia. Owing to concerns of metastatic calcification, the patient received an extensive workup, including a physical examination, hematologic and comprehensive metabolic labs, chest $\mathrm{x}$-ray, and $\mathrm{CT}$ of the abdomen and pelvis, which was negative for malignancy. He was found to be doing well with no evidence of local recurrence at one-year follow-up. The excised tissue consisted of a nodule of lamellar bone (Figure 2) adjacent to a lymphocytic infiltrate including follicles with germinal centers surrounded by mantle zones, scattered lymphocytes, plasma cells, and eosinophils. 
The second patient was a 46-year old male with a history of recurrent chalazion of the right upper eyelid that had been excised twice in the past. The patient was found to have a painless, $5 \times 6 \mathrm{~mm}$ whitish mass that had been present for approximately five years. He was otherwise healthy with no significant past medical history. Excisional biopsy revealed a firm, calcified mass with no evidence of sebaceous cell carcinoma or other malignancy. There was no evidence of recurrence at three months follow-up.

Histologic examination of the excised lesion showed nodular bone formation with osteoblastic rimming in the area of a sebaceous glands (Figure 3). There were histiocytes, cholesterol clefts, erythrocytes, and foreign body giant cells scattered throughout the field.

\section{DISCUSSION}

In general, cutaneous ossification is a rare entity that primarily affects the head and neck region. The majority of patients are Caucasian and female, with a peak incidence in the sixth decade. ${ }^{1}$ Etiologies can be primary or secondary. Primary causes include rare hereditary disorders, typically of congenital onset, such as Albright's hereditary osteodystrophy and progressive osseous heteroplasia. The term "osteoma cutis" refers to primary ossification of idiopathic origin. ${ }^{2}$ Secondary causes are typically due to a neoplasm or inflammation, including basal cell carcinoma, melanocytic nevi, chronic inflammatory acne, and sites of trauma or injection. ${ }^{2}$

Osseous metaplasia of the skin occurs when dermal cells are stimulated by local cellular factors to transform into bone-producing osteoblasts. Although the exact mechanism is unclear, it is thought that the presence of calcium and phosphorus in appropriate concentrations, $\mathrm{pH}$, oxygen tension, and certain enzymes are necessary for osteogenesis. ${ }^{1}$ Although there have been several cases of idiopathic osseous metaplasia occurring within breast $^{3}$, uterine ${ }^{4}$, bladder $^{5}$, and gastrointestinal ${ }^{6}$ tissue, there have been no previous reports of osseous metaplasia affecting the eyelid.

We believe that both of our patients exhibited secondary bone formation within the eyelid in conjunction with an antecedent or contemporary lesion. The eyelid lesion in our first patient was associated with lymphoid hyperplasia. The eyelid lesion in our second patient represents secondary ossification as a result of repeated surgical manipulation of the eyelid. Given the patient's history of recurrent chalazions that were surgically excised, it is likely that osseous metaplasia of fibroblasts or stromal stem cells occurred in response to trauma to the skin.

We distinguish the bone formation in our cases from the more common entity of subepidermal calcified nodules. ${ }^{7,8}$ Our two cases exhibited lamellar bone containing osteoclasts, Haversian canals and osteoblasts. Subepidermal calcified nodules contain globular calcium deposits beneath the epidermis.

An important differential diagnosis to consider for osseous metaplasia of the skin is nephrogenic systemic fibrosis (NSF), which occurs in patients with chronic kidney disease who have abnormalities of calcium/phosphate metabolism and are exposed to gadoliniumbased contrast agents. The presentation of NSF is a scleroderma-like systemic fibrosis with multi-organ involvement. ${ }^{9}$ In the skin, osseous metaplasia occurs in conjunction with characteristic diffuse brown-red macules. Neither of our patients had a history of kidney disease, rash, or evidence of systemic illness. Additionally, osseous metaplasia may occur in pilomatrixoma in the eyelid. ${ }^{10}$ There was no histologic evidence of pilomatrixoma in either of our two patients.

In conclusion, osseous metaplasia of the eyelid is a rare entity with a broad differential diagnosis, including both primary and secondary causes. Given its diverse etiologies, it is 
important for physicians to obtain relevant history concerning onset and progression, associated symptoms, and past medical history and to pursue appropriate laboratory and imaging tests in order to arrive at an accurate diagnosis. In both of our cases, treatment consisted of primary excision, which resulted in complete eradication of the lesion with no further recurrence.

\section{Acknowledgments}

Supported in part by departmental core grant NIH NEIP3006360 and an unrestricted departmental grant from Research to Prevent Blindness, Inc., New York, NY

\section{References}

1. Conlin PA, Jimenez-Quintero LP, Rapini RP. Osteomas of the skin revisited: a clinicopathologic review of 74 cases. The American Journal of Dermatopathology. 2002; 24(6):479-483. [PubMed: 12454599]

2. Burgdorf W, Nasemann T. Cutaneous osteomas: a clinical and histopathologic review. Archives of Dermatological Research. 1977; 260(2):121-135.

3. Ninomiya J, Oyama T, Horiguchi J, et al. Two case of breast cancer with cartilaginous and osseous metaplasia. Breast Cancer. 2005; 12(1):52-56. [PubMed: 15657524]

4. Umashankar T, Patted S, Handigund R. Endometrial osseous metaplasia: Clinicopathological study of a case and literature review. Journal of human reproductive sciences. 2010; 3(2):102. [PubMed: 21209755]

5. Eble J, Young R. Stromal osseous metaplasia in carcinoma of the bladder. The Journal of urology. 1991; 145(4):823. [PubMed: 1900893]

6. White V, Shaw AG, Tierney GM, et al. Osseous metaplasia in an ulcerating tubular adenoma of the colon: a case report. Journal of medical case reports. 2008; 2(1):1-3. [PubMed: 18182113]

7. Ferry AP. Subepidermal calcified nodules of the eyelid. Am J Ophthalmol. 1990; 110(1):316-318. [PubMed: 2396663]

8. Carvounis PE, Santi M, Stern JB, Miller M. Subepidermal calcified nodules. Ophthalmologica. 2005; 219(2):112-114. [PubMed: 15802937]

9. Wiedemeyer K, Kutzner H, Abraham JL, et al. The evolution of osseous metaplasia in localized cutaneous nephrogenic systemic fibrosis: a case report. The American journal of dermatopathology. 2009; 31(7):674. [PubMed: 19633532]

10. Rizvi SA, Naim M, Alam MS. A giant upper eyelid ossifying pilomatrixoma. Indian j Ophthalmol. 2008; 56(6):509-511. [PubMed: 18974526] 


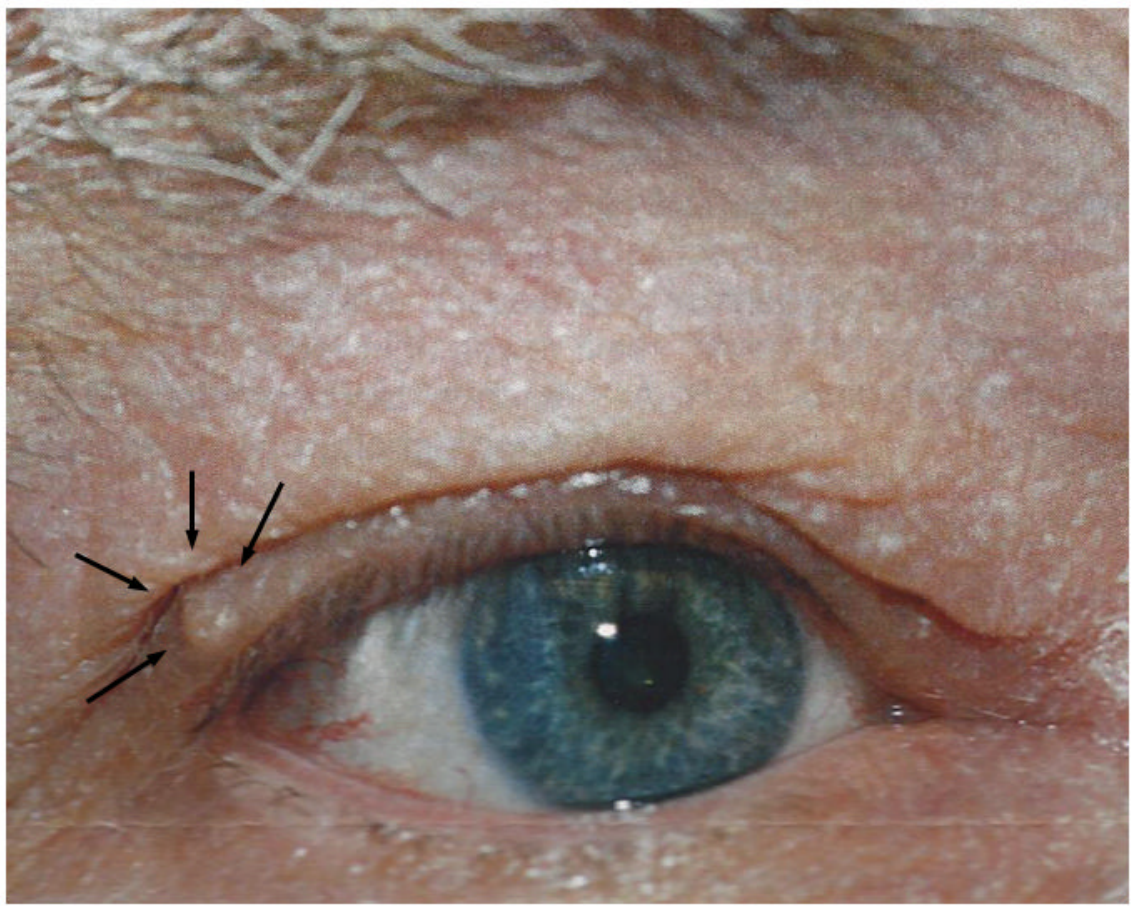

Figure 1.

A firm nodule is present on the lateral aspect of the right upper lid (arrows). 


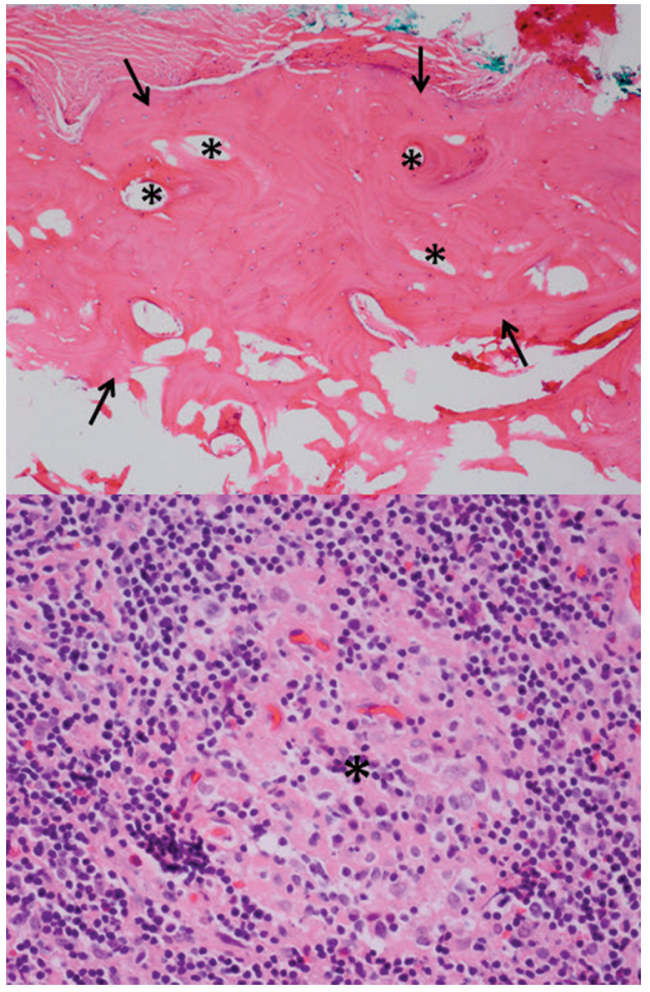

Figure 2.

Top. The tumor consists of ossified, calcified lamellar bone (arrows) that is beginning to develop a Haversian canal system (asterisks). Bottom. The lesion also contains an area of lymphoid hyperplasia consisting of follicles (*) surround by small, round lymphocytes. (hematoxylin-eosin, top, 25X, bottom, 100X). 


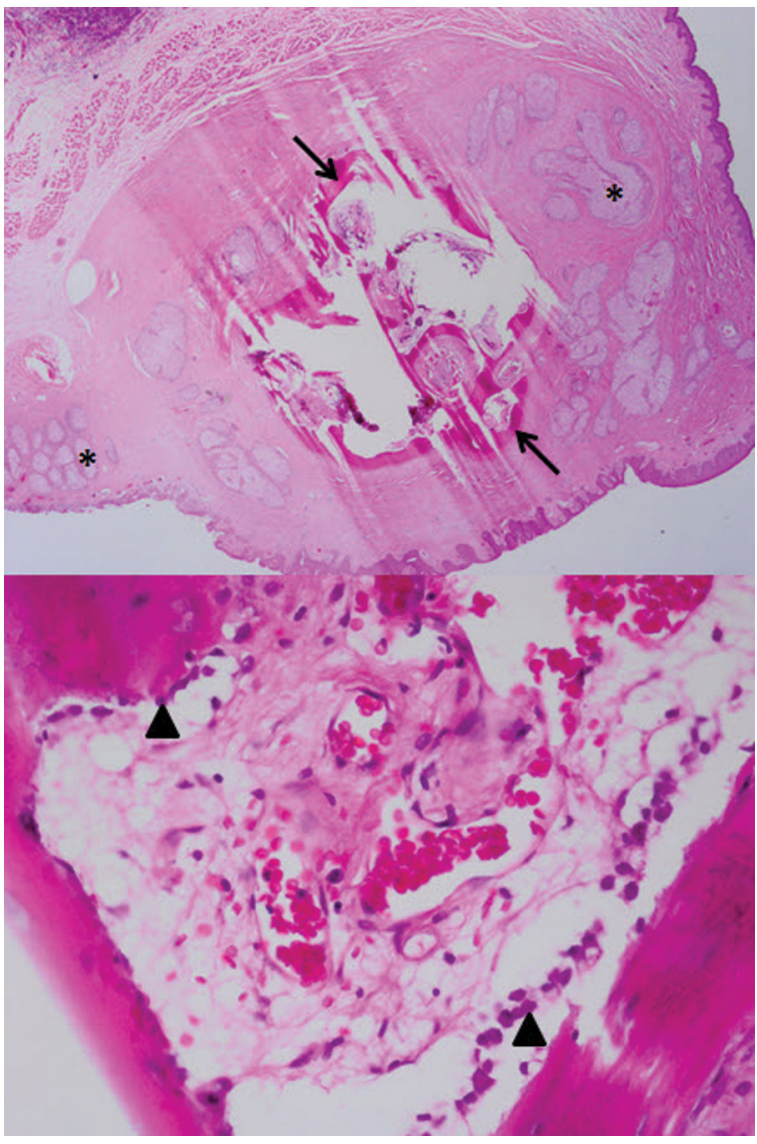

Figure 3 .

Top. The tarsal plate contains Meibomian glands $(*)$ and is expanded by a nodule of lamellar bone (between arrows). Bottom. The bone exhibits osteoblastic rimming (arrowheads). (hematoxylin-eosin, top 5X, bottom 100X). 